\title{
PAPER \\ On the Bit Error Probability of OFDM and FBMC-OQAM Systems in Rayleigh and Rician Multipath Fading Channels
}

\author{
Liming $\mathrm{LI}^{\dagger \mathrm{a})}$, Student Member, Yang $\mathrm{WANG}^{\dagger \mathrm{b})}$, and Liqin DING ${ }^{\dagger \mathrm{c})}$, Nonmembers
}

\begin{abstract}
SUMMARY Filter bank multicarrier with offset quadrature amplitude modulation (FBMC-OQAM) is considered an alternative to conventional orthogonal frequency division multiplexing (OFDM) to meet the various requirements proposed by future communication networks. Among the different perspectives on the merits of FBMC-OQAM and OFDM, a straightforward metric is the bit error probability (BEP). This paper presents a general analytical framework for BEP evaluation that is applicable to FBMCOQAM and OFDM systems in both Rayleigh and Rician multipath fading channels. Explicit BEP expressions are derived for Gray-coded pulse amplitude modulation (PAM) and square quadrature amplitude modulation (QAM) signals with arbitrary constellation sizes. The theoretical analysis results show excellent agreement with the numerical simulation results in different channel scenarios.

key words: bit error probability, FBMC-OQAM, OFDM, multipath channel, Gray coding
\end{abstract}

\section{Introduction}

As a multicarrier technique, orthogonal frequency division multiplexing (OFDM) has been extensively used in many communication systems, such as Long Term Evolution (LTE), Wireless Local Area Networks (WLANs) and Digital Video Broadcasting-Terrestrial (DVB-T). Using the cyclic prefix $(\mathrm{CP})$ as a guard interval against intersymbol interference (ISI), CP-OFDM offers good immunity to the frequency selectivity of channels. However, this comes at a price: an increased sensitivity to synchronization offsets and the Doppler spread, and also a poor spectrum localization property. This prevents OFDM from being an efficient waveform technology in some new emerging communication scenarios.

In recent years, the filter bank multicarrier (FBMC) was proposed as an important candidate waveform technique to efficiently support the asynchronous and heterogeneous network scenarios expected in the future [1], such as massive machine-type communications (mMTC) [2], coordinated multipoint (CoMP) [3], cognitive radio (CR) [4], and deviceto-device (D2D) communications [5]. As FBMC uses filters with good time-frequency localization (TFL) property [6], it is robust against synchronization errors [7] and offers much better spectrum performance than OFDM [8]. One

Manuscript received December 6, 2017.

Manuscript revised February 26, 2019.

Manuscript publicized June 17, 2019.

${ }^{\dagger}$ The authors are with the Shenzhen Graduate School, Harbin Institute of Technology, Shenzhen, 518055, China.

a)E-mail: limingli@aliyun.com

b) E-mail: yangw@ @it.edu.cn (Corresponding author)

c) E-mail: dingliqin@ @it.edu.cn

DOI: 10.1587/transcom.2017EBP3451 notable FBMC variant is the FBMC with offset quadrature amplitude modulation (FBMC-OQAM). Different from the conventional OFDM that transmits complex-valued quadrature amplitude modulation (QAM) symbols at a given symbol rate, FBMC-OQAM transmits real-valued pulse amplitude modulated (PAM) symbols at twice this symbol rate, with the orthogonality of the waveform restricted only to the real field [1]. By virtue of this modulation type, FBMCOQAM achieves the highest spectral efficiency as a non-CPOFDM system while maintaining good TFL property, and provides superior performance to OFDM in some frequencydispersive channels [9] and asynchronous scenarios [10].

The bit error probability (BEP) performances of FBMC-OQAM and OFDM systems have been compared by means of numerical simulations in a great number of studies [11]-[13]. Several other papers proposed theoretical analysis methods under different conditions. A theoretical BEP performance evaluation of the downlink of asynchronous OFDM and FBMC-OQAM cellular radio communication systems was provided by [14]. Additionally, a theoretical BEP study of OFDM and FBMC-OQAM systems considering the nonlinear distortion of the amplifier was introduced by [15] and [16]. Moreover, the closed-form expressions of the BEP for OFDM and FBMC-OQAM systems with pilot-aided channel estimation were derived by [17]. All of these works focused on Rayleigh flat-fading or time-invariant frequency-selective channels. [9] extended the work of [17] to the doubly-selective channels, but still, the analysis applies only to the Rayleigh channels. In [18], the BEP performances of FBMC system over Rayleigh and Nakagami-m channels were investigated in the form of asymptotic analytical expressions, based on the analysis of the signal interference noise ratio (SINR).

The Rician channel is another important and more general channel model by the truth that there is likely to be a direct path component, i.e., the line of sight (LOS) path, in many practical environments. This phenomenon is strengthened with the trend toward higher frequency band usage [19] and in some specific communication scenarios expected in the future, such as D2D communications, where the beam width of the antenna and the distance between the transceivers may both be much limited [20], [21]. Therefore, in this study we aim to develop a general analytical framework for BEP evaluation that covers both Rayleigh and Rician fading, flat and doubly-selective channels, and FBMC-OQAM and OFDM systems. It should be noted that in this study we only consider single-tap equalization, which 
is most widely used as a trade-off between the complexity and performance [8], [9]. The channel is assumed to be estimated perfectly.

The remainder of this paper is organized as follows. Section 2 describes a unified baseband model of OFDM and FBMC-OQAM systems. A general analytical framework for the BEP calculation of PAM and QAM signals in Rayleigh and Rician multipath channels are presented in Sect. 3. The validation simulation results and discussion are presented in Sect. 4. Finally, the conclusions and future works are drawn in Sect. 5.

\section{System Model}

\subsection{Unified Discrete-Time Baseband Model}

The transmitted baseband signal $s(k)$, consisting of $M$ subcarriers during $N$ symbol periods, for both OFDM and FBMC systems can be expressed as [1]

$$
s(k)=\sum_{n=1}^{N} \sum_{m=1}^{M} x_{m, n} p_{m, n}(k)
$$

where indices $(m, n)$ refers to the $m$-th subcarrier and the $n$-th symbol, $x_{m, n}$ is the data symbol to be transmitted, and $p_{m, n}(k)$ is the corresponding synthesis filter signal modulated on $x_{m, n}$.

Note that $x_{m, n}$ is generally a complex QAM symbol in OFDM but a real PAM symbol in FBMC-OQAM systems. For non-CP-OFDM, the synthesis filter signal $p_{m, n}(k)$ is given by

$$
p_{m, n}(k)=p\left(k / f_{s}-n T\right) e^{j 2 \pi m F\left(k-n T f_{s}\right) / f_{s}}
$$

where $p(k)$, called the prototype filter, is a rectangular window function signal, $T$ and $F$, which satisfy $T F=1$, are the symbol period and subcarrier bandwidth respectively, $f_{s}$ denotes the sampling rate, and $j$ is the imaginary unit in this paper. For FBMC-OQAM the synthesis filter signal is slightly different from that of OFDM, as follows:

$$
p_{m, n}(k)=e^{j \theta_{m, n}} p\left(k / f_{s}-n T\right) e^{j 2 \pi m F\left(k-n T f_{s}\right) / f_{s}}
$$

where prototype filter $p(k)$ is usually a real symmetric filter with good TFL property, e.g., PHYDYAS [22], [23], Hermite [24], and IOTA [25] filters, $\theta_{m, n}=\frac{\pi}{2}(m+n)$ is the phase shift required to fulfill the real orthogonality condition for OQAM modulation. The symbol period of FBMC-OQAM is half that of OFDM to achieve the same bandwidth efficiency, i.e., $T F=\frac{1}{2}$.

Considering a time-variant multipath fading channel model in terms of channel filter taps, the received signal at the receiver can be represented as

$$
r(k)=\sum_{l=0}^{L} h_{l}(k) s\left(k-\tau_{l}\right)+\omega(k)
$$

where $h_{l}(k)$ denotes the $l$-th channel tap with delay $\tau_{l}$ of total $L+1$ at time $k$, and $\omega(k) \sim \mathcal{C N}\left(0, P_{\omega}\right)$ denotes the white Gaussian noise. Moreover, $\left\{h_{l}(k)\right\}_{k}$ is modeled as circular symmetric processes independent across the taps. For a Rayleigh channel, $h_{l}(k)=\sqrt{P_{l}} z_{l}(k)$ is regarded as a complex Gaussian variable $z_{l}(k) \sim \mathcal{C N}(0,1)$ weighted by $\sqrt{P_{l}}$, the strength of this diffused path. In the case of a Rician channel, there is an LOS component that is usually contained in the first tap, and then $h_{0}(k)=\sqrt{P_{L O S}}+\sqrt{P_{0}} z_{0}(k)$, where $P_{\text {LOS }}$ represents the strength of the LOS component [26, ANNEX 2]. For simplicity, all taps are normalized such that the expected value of the path gains' total power is 1 .

By performing filtering and down-sampling to the received signal $r(k)$ at the receiver, we can obtain the $(m, n)$-th filtered signal $y_{m, n}$ :

$$
y_{m, n}=\left\langle r(k), q_{m, n}(k)\right\rangle=\sum_{k} r(k) q_{m, n}^{*}(k)
$$

where $q_{m, n}(k)$ is the corresponding analysis filter signal, and \langle\rangle denotes the inner product.

After the single-tap equalization, the decision statistic $x_{m, n}^{\prime}$ of the $(m, n)$-th data symbol is obtained:

$$
x_{m, n}^{\prime}=\frac{y_{m, n}}{h_{m, n}^{\prime}}=\frac{\left\langle r(k), q_{m, n}(k)\right\rangle}{h_{m, n}^{\prime}}
$$

where

$$
h_{m, n}^{\prime}=\sum_{l=0}^{L}\left\langle p_{m, n}\left(k-\tau_{l}\right) h_{l}(k), q_{m, n}(k)\right\rangle
$$

represents the equivalent channel coefficient corresponds to the $(m, n)$-th data symbol after filtering operations.

\subsection{Matrix Form of the Baseband Model}

For convenience, we represent the baseband system model in matrix form in the following as in [8], [9]. Let $\mathbf{s}=$ $[s(1), s(2), \ldots, s(K)]^{T}$ denote the transmitted signal vector, with $K=N T f_{s}$ being the total number of samples during $N$ symbol periods, $\mathbf{x}=\left[x_{1,1}, x_{2,1}, \ldots, x_{M, 1}, x_{1,2}, \ldots, x_{M, N}\right]^{T}$ denote the vectorized forms of the data symbols, $\mathbf{P}=$ $\left[\mathbf{p}_{1,1}, \mathbf{p}_{2,1}, \ldots, \mathbf{p}_{M, 1}, \mathbf{p}_{1,2}, \ldots, \mathbf{p}_{M, N}\right]$ denote the corresponding synthesis filter signal matrix with each element vector $\mathbf{p}_{m, n}=\left[p_{m, n}(1), p_{m, n}(2), \ldots, p_{m, n}(K)\right]^{T}$ representing the signal vector of $p_{m, n}(k)$, and $\operatorname{vec}()$ denote the vectorization operator. (1) can be rewritten as

$$
\mathbf{S}=\mathbf{P} \mathbf{x}=\mathbf{P} \operatorname{vec}(\mathbf{X})
$$

where

$$
\mathbf{X}=\left(\begin{array}{cccc}
x_{1,1} & x_{1,2} & \ldots & x_{1, N} \\
x_{2,1} & x_{2,2} & \ldots & x_{2, N} \\
\vdots & \vdots & \ddots & \vdots \\
x_{M, 1} & x_{M, 2} & \ldots & x_{M, N}
\end{array}\right)
$$


Similarly, let $\mathbf{Q}=\left[\mathbf{q}_{1,1}, \mathbf{q}_{2,1}, \ldots, \mathbf{q}_{M, 1}, \mathbf{q}_{1,2}, \ldots, \mathbf{q}_{M, N}\right]$ denote the analysis filter signal matrix, and $\omega$ and $\omega^{\prime}$ denote the noise signal vector before and after filtering, respectively. We can obtain the received signal vector $\mathbf{r}=[r(1), r(2), \ldots, r(K)]^{T}$ and the filtered signal vector $\mathbf{y}=\left[y_{1,1}, y_{2,1}, \ldots, y_{M, 1}, y_{1,2}, \ldots, y_{M, N}\right]^{T}$ as

$$
\begin{aligned}
& \mathbf{r}=\mathbf{H s}+\boldsymbol{\omega} \\
& \mathbf{y}=\mathbf{Q}^{H} \mathbf{r}=\mathbf{Q}^{H} \mathbf{H} \mathbf{s}+\omega^{\prime}
\end{aligned}
$$

where

$$
\mathbf{H}=\left(\begin{array}{cccccccc}
h_{\tau_{0}}(1) & 0 & \ldots & \ldots & \ldots & \ldots & 0 & 0 \\
h_{\tau_{1}}(1) & h_{\tau_{0}}(2) & 0 & \ldots & \ldots & \ldots & \ldots & 0 \\
\vdots & h_{\tau_{1}}(2) & \ddots & \ddots & \ldots & \ldots & \vdots & \vdots \\
h_{\tau_{L}}(1) & \vdots & \ddots & \ddots & 0 & \ldots & \vdots & \vdots \\
0 & h_{\tau_{L}}(2) & \vdots & \ddots & h_{\tau_{0}}(K-L) & \ddots & \vdots & \vdots \\
0 & 0 & \ddots & \vdots & h_{\tau_{1}}(K-L) & \ddots & 0 & \vdots \\
\vdots & \vdots & \ddots & \ddots & \vdots & \ddots & h_{\tau_{0}}(K-1) & 0 \\
0 & 0 & \ldots & & h_{\tau_{L}}(K-L) & \ldots & h_{\tau_{1}}(K-1) & h_{\tau_{0}}(K)
\end{array}\right)
$$

is the channel convolution matrix and and the first two taps are assumed to be adjacent in samples to show this matrix briefly. Then $y_{m, n}$ and $h_{m, n}^{\prime}$ in (6) can be calculated as

$$
\begin{aligned}
& y_{m, n}=\mathbf{q}_{m, n}^{H} \mathbf{r}=\mathbf{q}_{m, n}^{H} \mathbf{H P x}+\omega_{m, n}^{\prime} \\
& h_{m, n}^{\prime}=\mathbf{q}_{m, n}^{H} \mathbf{H} \mathbf{p}_{m, n}
\end{aligned}
$$

Note that $\mathbf{Q}$ generally equals $\mathbf{P}$ in FBMC and nonCP-OFDM systems, and the perfect reconstruction property of the prototype filters leads to $\mathbf{Q}^{H} \mathbf{P}=\mathbf{I}_{M N}$ for OFDM and $\mathfrak{R}\left\{\mathbf{Q}^{H} \mathbf{P}\right\}=\mathbf{I}_{M N}$ for FBMC-OQAM, where $\mathbf{I}_{M N}$ is the $M N \times M N$ identity matrix. In the CP-OFDM case, $\mathrm{CP}$ can be implemented by adding the corresponding signal samples and zero samples at the beginning of $\mathbf{p}_{\mathbf{m}, \mathbf{n}}$ and $\mathbf{q}_{\mathbf{m}, \mathbf{n}}$, respectively.

In addition, the synchronization errors, timing offset (TO), and carrier frequency offset (CFO) can be presented easily by performing some specific matrix operations on Q. For example, for all subcarriers, TO with value $\varepsilon$ can be involved by shifting $\mathbf{Q}$ by $f_{s} \varepsilon$ samples in the column dimension, and CFO with value $\rho$ can be involved by using $\left(\rho \otimes \mathbf{1}_{1 \times M N}\right) \circ \mathbf{Q}$ as the new analysis filter signal matrix $\mathbf{Q}$, where $\rho=\left[e^{j 2 \pi \rho / f_{s}}, e^{j 2 \pi 2 \rho / f_{s}}, \ldots, e^{j 2 \pi k \rho / f_{s}}, \ldots, e^{j 2 \pi K \rho / f_{s}}\right]$, and $\mathbf{1}_{1 \times M N}$ denotes the $1 \times M N$ matrix whose entries are all ones, and $\circ$ denotes the Hadamard product.

\section{BEP Statistical Analysis in Rician and Rayleigh Mul- tipath Channels}

As mentioned in the previous sections, at the receiver, the decision statistic $x_{m, n}^{\prime}$ is determined to be a PAM symbol in FBMC-OQAM systems or a QAM symbol in OFDM systems. In this study we only consider the Gray coding, which is the most common case in communication systems. Based on the unified baseband model introduced in Sect. 2, we propose a general analytical framework for BEP calculations applicable to both FBMC-OQAM and OFDM systems in Rayleigh and Rician multipath fading channels. Because a square QAM signal can be regarded as a combination of two independent PAM signals, as shown in Fig. 1, we begin with the BEP analysis for the PAM signal. Firstly, let us consider the 4-PAM case.

\subsection{BEP Analysis for 4-PAM Signal}

Figure 1(a) shows the constellation of the 4-PAM signal and its decision boundaries. Each signal point from the set $\{-3 d,-d, d, 3 d\}$ represents a 2-bit symbol formatted as $b 1 b 2$, and $d$ equals half the distance between two adjacent signal points. The receiver determines the value of each bit according to the real part of $x_{m, n}^{\prime}$, and the decision rules can be described in a straightforward manner:

$$
\begin{aligned}
& \text { if } \mathfrak{R}\left\{x_{m, n}^{\prime}\right\} \geq 0 \text { then } b 1=0 \\
& \text { else } b 1=1 \\
& \text { if }-2 d \leq \mathfrak{R}\left\{x_{m, n}^{\prime}\right\}<2 d \text { then } b 2=0 \\
& \text { else } b 2=1
\end{aligned}
$$

Thus, the BEPs of bits $b 1$ and $b 2, P_{b 1}$ and $P_{b 2}$, and the average BEP of the 4-PAM signal, $P_{b}$, can be calculated as

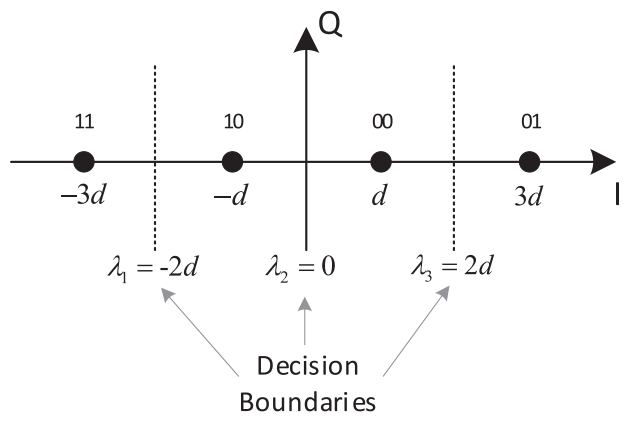

(a) 4-PAM

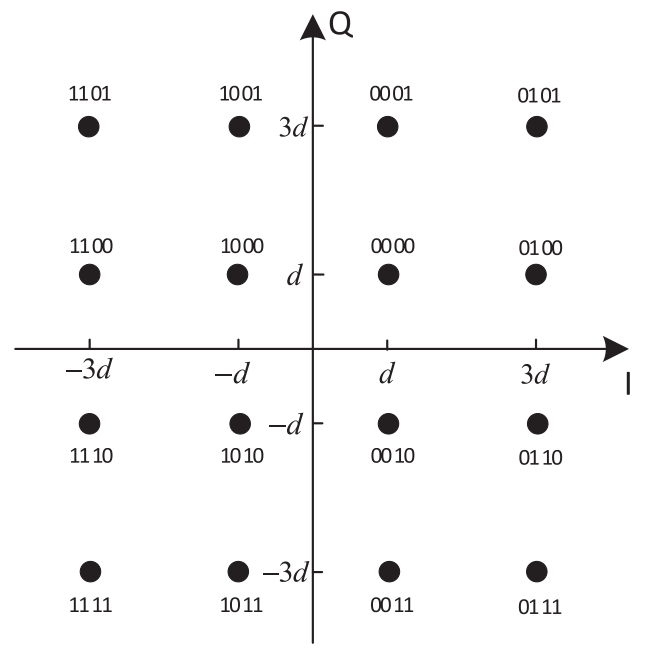

(b) 16-QAM

Fig. 1 Constellations of 4-PAM and 16-QAM Signals with Gray coding. 


$$
\begin{aligned}
P_{b 1}= & \frac{1}{2} \operatorname{Re}\left\{\mathfrak{R}\left\{x_{m, n}^{\prime}\right\}<0 \mid x_{m, n}=d, 3 d\right\} \\
& +\frac{1}{2} \operatorname{Re}\left\{\mathfrak{R}\left\{x_{m, n}^{\prime}\right\} \geq 0 \mid x_{m, n}=-d,-3 d\right\} \\
P_{b 2}= & \frac{1}{4} \operatorname{Re}\left\{\begin{array}{l}
\mathfrak{R}\left\{x_{m, n}^{\prime}\right\} \geq 2 d \text { or } \\
\mathfrak{R}\left\{x_{m, n}^{\prime}\right\}<-2 d
\end{array} \mid x_{m, n}= \pm d\right\} \\
& +\frac{1}{4} \operatorname{Re}\left\{-2 d \leq \mathfrak{R}\left\{x_{m, n}^{\prime}\right\}<2 d \mid x_{m, n}= \pm 3 d\right\} \\
P_{b}= & \frac{1}{2}\left(P_{b 1}+P_{b 2}\right)
\end{aligned}
$$

For convenience, we define $F_{\mathrm{Re}, x_{m, n}^{\prime}}\left(\lambda, x_{m, n}\right)$ to be the conditional cumulative distribution function (CDF) of $\mathfrak{R}\left\{x_{m, n}^{\prime}\right\}$ :

$$
F_{\mathrm{Re}, x_{m, n}^{\prime}}\left(\lambda, x_{m, n}\right)=\operatorname{Pr}\left\{\mathfrak{R}\left\{x_{m, n}^{\prime}\right\}<\lambda \mid x_{m, n}\right\}
$$

where $\lambda$ denotes the decision boundary. Accordingly, $\operatorname{Pr}\left\{\operatorname{Re}\left\{x_{m, n}^{\prime}\right\} \geq \lambda \mid x_{m, n}\right\}=1-F_{\operatorname{Re}, x_{m, n}^{\prime}}\left(\lambda, x_{m, n}\right)$, and (15) and (16) can be calculated if the values of $F_{\operatorname{Re}, x_{m, n}^{\prime}}\left(\lambda, x_{m, n}\right)$ are obtained for the corresponding $\lambda$ and $x_{m, n}$.

Considering the presence of ISI and ICI, $F_{\mathrm{Re}, x_{m, n}^{\prime}}$ $\left(\lambda, x_{m, n}\right)$ refers to not only $x_{m, n}$ but also the other data symbols in vector $\mathbf{x}$. Although treating the ISI and ICI as Gaussian noise is a more simplified method, this will cause observable approximate errors in some doubly-selective channels at high SNRs [8], [9]. According to $\mathbf{x}=\mathbf{G}\left[\mathbf{x}_{I}^{T}, x_{m . n}\right]^{T}$, where $\mathbf{G}$ is a permutation matrix used to split the transmitted symbols $\mathbf{x}$ into interfering symbols $\mathbf{x}_{I}$ and the symbol of interest, $x_{m, n}$, a numerical calculation process as in [9] should be performed:

$$
F_{\mathrm{Re}, x_{m, n}^{\prime}}\left(\lambda, x_{m, n}\right)=\frac{1}{C} \sum_{\mathbf{x}_{I} \in \mathbb{S}} F_{\mathrm{Re}, x_{m, n}^{\prime}}\left(\lambda, \mathbf{G}\left[\mathbf{x}_{I}^{T}, x_{m . n}\right]^{T}\right)
$$

where $F_{\mathrm{Re}, x_{m, n}^{\prime}}\left(\lambda, \mathbf{G}\left[\mathbf{x}_{I}^{T}, x_{m . n}\right]^{T}\right)$ is the probability function conditioned on the data symbol vector $\mathbf{x}=\mathbf{G}\left[\mathbf{x}_{I}^{T}, x_{m . n}\right]^{T}, \mathbb{S}$ is the set of $\mathbf{x}_{I}$, and $C$ is the cardinality of $\mathbb{S}$. A proper size of $\mathbb{S}$ should be chosen to as a trade-off between the computation complexity and accuracy.

If the statistic characteristics of $x_{m, n}^{\prime}$ conditioned on the transmitted data symbols $\mathbf{x}$ are known, then $F_{\mathrm{Re}, x_{m, n}^{\prime}}(\lambda, \mathbf{x})$ can be calculated easily as a marginal distribution function of $x_{m, n}^{\prime}$. For Rayleigh and Rician fading channels, given the transmitted data symbols $\mathbf{x}, y_{m, n}$ and $h_{m, n}^{\prime}$ can be considered as a linear combination of Gaussian random variables, according to (13) and (14). This means that $y_{m, n}$ and $h_{m, n}^{\prime}$ are a pair of correlated complex-valued Gaussian random variables, and their expectations can be calculated as

$$
\begin{aligned}
\mathbb{E}\left[y_{m, n} \mid \mathbf{x}\right] & =\mathbf{q}_{m, n}^{H} \mathbb{E}[\mathbf{H}] \mathbf{P x}+\mathbb{E}\left[\omega_{m, n}^{\prime}\right] \\
& = \begin{cases}0 & \text { Rayleigh channels, } \\
\sqrt{P_{L O S}}\left(x_{m, n}+j I_{i n t}\right) & \text { Rician channels. }\end{cases}
\end{aligned}
$$

$$
\begin{aligned}
\mathbb{E}\left[h_{m, n}^{\prime}\right] & =\mathbf{q}_{m, n}^{H} \mathbb{E}[\mathbf{H}] \mathbf{p}_{m, n} \\
& = \begin{cases}0 & \text { Rayleigh channels }, \\
\sqrt{P_{L O S}} & \text { Rician channels. }\end{cases}
\end{aligned}
$$

where $j I_{\text {int }}$ denotes the intrinsic imaginary interference caused by other data symbols in $\mathbf{x}$ in FBMC-OQAM systems and equals zero in OFDM systems.

In the case of Rayleigh fading channels, $y_{m, n}$ and $h_{m, n}^{\prime}$ are zero-mean, and then $x_{m, n}^{\prime}$, given by $y_{m, n} / h_{m, n}^{\prime}$, follows a complex ratio distribution defined in [27] with CDF given by $(\mathrm{A} \cdot 1)$ in Appendix.

In the case of Rician fading, however, according to (20) and (21), $y_{m, n}$ and $h_{m, n}^{\prime}$ are not zero-mean because of the LOS component, and then the CDF of complex ratio distribution derived in [9] and [27] can no longer be applied. Thus, we introduce another method to calculate $F_{\mathrm{Re}, x_{m, n}^{\prime}}(\lambda, \mathbf{x})$ in the following.

Firstly, $F_{\operatorname{Re}, x_{m, n}^{\prime}}(\lambda, \mathbf{x})$ can be written as

$$
\begin{aligned}
& F_{\operatorname{Re}, x_{m, n}^{\prime}}(\lambda, \mathbf{x}) \\
& =\operatorname{Pr}\left\{\mathfrak{R}\left\{\frac{y_{m, n} h_{m, n}^{\prime *}}{\left|h_{m, n}^{\prime}\right|^{2}}\right\}<\lambda \mid \mathbf{x}\right\} \\
& =\operatorname{Pr}\left\{\mathfrak{R}\left\{h_{m, n}^{\prime *}\left(y_{m, n}-\lambda h_{m, n}^{\prime}\right)\right\}<0 \mid \mathbf{x}\right\}
\end{aligned}
$$

Namely, $F_{\operatorname{Re}, x_{m, n}^{\prime}}(\lambda, \mathbf{x})$ is equivalent to the conditional probability of $\mathfrak{R}\left\{h_{m, n}^{\prime *}\left(y_{m, n}-\lambda h_{m, n}^{\prime}\right)\right\}<0$, and $\mathfrak{R}\left\{h_{m, n}^{\prime *}\left(y_{m, n}-\right.\right.$ $\left.\left.\lambda h_{m, n}^{\prime}\right)\right\}$ can be regarded as a Hermitian quadratic form variable: $\frac{1}{2}\left(\alpha_{m, n}^{*} \beta_{m, n}+\alpha_{m, n} \beta_{m, n}^{*}\right)$, where $\alpha_{m, n}=h_{m, n}^{\prime}$ and $\beta_{m, n}=y_{m, n}-\lambda h_{m, n}^{\prime}$. As described in previous, $y_{m, n}$ and $h_{m, n}^{\prime}$ are Gaussian distributed, and thus $\frac{1}{2}\left(\alpha_{m, n}^{*} \beta_{m, n}+\right.$ $\left.\alpha_{m, n} \beta_{m, n}^{*}\right)$ is a Gaussian Hermitian quadratic form variable. Then, the conditional probability in (22) can be obtained as an extension to the result in [28] and [29]:

$$
F_{\mathrm{Re}, x_{m, n}^{\prime}}(\lambda, \mathbf{x})=Q(a, b)-\frac{v_{2}}{v_{1}+v_{2}} I_{0}(a b) e^{-\frac{a^{2}+b^{2}}{2}}
$$

where $Q(a, b)$ is the Marcum $Q$ function, $I_{0}(\cdot)$ is the 0 -th order modified Bessel function of the first kind, and

$$
\left\{\begin{aligned}
a & =\sqrt{2 v_{1}^{2} v_{2}\left(u_{1} v_{2}-u_{2}\right) /\left(v_{1}+v_{2}\right)^{2}} \\
b & =\sqrt{2 v_{1} v_{2}^{2}\left(u_{1} v_{1}+u_{2}\right) /\left(v_{1}+v_{2}\right)^{2}} \\
u_{1} & =\frac{1}{4}\left[\left|m_{\alpha}\right|^{2} m_{\beta \beta}+\left|m_{\beta}\right|^{2} m_{\alpha \alpha}-2 \Re\left\{m_{\alpha}^{*} m_{\beta} m_{\alpha \beta}\right\}\right] \\
u_{2} & =\Re\left\{m_{\alpha}^{*} m_{\beta}\right\} \\
v_{1} & =\sqrt{\omega^{2}+4 /\left(m_{\alpha \alpha} m_{\beta \beta}-\left|m_{\alpha \beta}\right|^{2}\right)}-\omega \\
v_{2} & =\sqrt{\omega^{2}+4 /\left(m_{\alpha \alpha} m_{\beta \beta}-\left|m_{\alpha \beta}\right|^{2}\right)}+\omega \\
\omega & =2 \mathfrak{R}\left\{m_{\alpha \beta}\right\} /\left(m_{\alpha \alpha} m_{\beta \beta}-\left|m_{\alpha \beta}\right|^{2}\right)
\end{aligned}\right.
$$

where $m_{\alpha}, m_{\beta}, m_{\alpha \alpha}$ and $m_{\beta \beta}$ are the means and variances of $\alpha$ and $\beta$ respectively, and $m_{\alpha \beta}$ denotes the covariance of $\alpha$ and $\beta$. These moments can be calculated 


$$
\left\{\begin{array}{l}
m_{\alpha}=\mathbb{E}\left[\alpha_{m, n} \mid \mathbf{x}\right]=\mathbb{E}\left[h_{m, n}^{\prime}\right]=\sqrt{P_{L O S}} \mathbf{q}_{m, n}^{H} \mathbf{p}_{m, n} \\
m_{\beta}=\mathbb{E}\left[\beta_{m, n} \mid \mathbf{x}\right]=\mathbb{E}\left[\left(y_{m, n}-\lambda h_{m, n}^{\prime}\right) \mid \mathbf{x}\right]=\sqrt{P_{L O S}} \mathbf{q}_{m, n}^{H} \mathbf{P} \mathbf{x}-\lambda m_{\alpha} \\
m_{\alpha \alpha}=\mathbb{E}\left[\left(\alpha_{m, n}-m_{\alpha}\right)\left(\alpha_{m, n}-m_{\alpha}\right)^{*} \mid \mathbf{x}\right]=\mathbb{E}\left[\left|h_{m, n}^{\prime}\right|^{2}\right]-\left|m_{\alpha}\right|^{2} \\
m_{\beta \beta}=\mathbb{E}\left[\left(\beta_{m, n}-m_{\beta}\right)\left(\beta_{m, n}-m_{\beta}\right)^{*} \mid \mathbf{x}\right]=\mathbb{E}\left[\left|y_{m, n}\right|^{2} \mid \mathbf{x}\right]+\lambda^{2} \mathbb{E}\left[\left|h_{m, n}^{\prime}\right|^{2}\right]-2 \lambda \Re\left\{\mathbb{E}\left[y_{m, n} h_{m, n}^{\prime *} \mid \mathbf{x}\right]\right\}-\left|m_{\beta}\right|^{2} \\
m_{\alpha \beta}=\mathbb{E}\left[\left(\alpha_{m, n}-m_{\alpha}\right)\left(\beta_{m, n}-m_{\beta}\right)^{*} \mid \mathbf{x}\right]=\mathbb{E}\left[y_{m, n}^{*} h_{m, n}^{\prime} \mid \mathbf{x}\right]-\lambda \mathbb{E}\left[\left|h_{m, n}^{\prime}\right|^{2}\right]-m_{\alpha} m_{\beta}^{*}
\end{array}\right.
$$

where

$$
\left\{\begin{array}{l}
\mathbb{E}\left[\left|y_{m, n}\right|^{2} \mid \mathbf{x}\right]=\mathbb{E}\left[\left(\mathbf{x}^{T} \operatorname{vec}\left(\mathbf{q}_{m, n}^{H} \mathbf{H P}\right)\right)\left(\operatorname{vec}\left(\mathbf{q}_{m, n}^{H} \mathbf{H P}\right)^{H} \mathbf{x}^{*}\right)\right]+P_{\omega} \\
=\mathbb{E}\left[\mathbf{x}^{T}\left(\mathbf{P}^{T} \otimes \mathbf{q}_{m, n}^{H}\right) \operatorname{vec}(\mathbf{H}) \operatorname{vec}(\mathbf{H})^{H}\left(\mathbf{P}^{T} \otimes \mathbf{q}_{m, n}^{H}\right)^{H} \mathbf{x}^{*}\right]+P_{\omega}=\mathbf{x}^{T}\left(\mathbf{P}^{T} \otimes \mathbf{q}_{m, n}^{H}\right) \mathbf{R}_{\mathrm{vec}(\mathbf{H})}\left(\mathbf{P}^{T} \otimes \mathbf{q}_{m, n}^{H}\right)^{H} \mathbf{x}^{*}+P_{\omega} \\
\mathbb{E}\left[\left|h_{m, n}^{\prime}\right|^{2}\right]=\mathbb{E}\left[\left(\mathbf{q}_{m, n}^{H} \mathbf{H} \mathbf{p}_{m, n}\right)\left(\mathbf{q}_{m, n}^{H} \mathbf{H} \mathbf{p}_{m, n}\right)^{H}\right]=\left(\mathbf{p}_{m, n}^{T} \otimes \mathbf{q}_{m, n}^{H}\right) \mathbf{R}_{\mathrm{vec}(\mathbf{H})}\left(\mathbf{p}_{m, n}^{T} \otimes \mathbf{q}_{m, n}^{H}\right)^{H} \\
\mathbb{E}\left[y_{m, n} h_{m, n}^{\prime *} \mid \mathbf{x}\right]=\mathbf{x}^{T}\left(\mathbf{P}^{T} \otimes \mathbf{q}_{m, n}^{H}\right) \mathbf{R}_{\mathrm{vec}(\mathbf{H})}\left(\mathbf{p}_{m, n}^{T} \otimes \mathbf{q}_{m, n}^{H}\right)^{H} \\
\mathbb{E}\left[y_{m, n}^{*} h_{m, n}^{\prime} \mid \mathbf{x}\right]=\mathbb{E}\left[\left(y_{m, n} h_{m, n}^{\prime *}\right)^{*} \mid \mathbf{x}\right]=\left(\mathbf{p}_{m, n}^{T} \otimes \mathbf{q}_{m, n}^{H}\right) \mathbf{R}_{\mathrm{vec}(\mathbf{H})}\left(\mathbf{P}^{T} \otimes \mathbf{q}_{m, n}^{H}\right)^{H} \mathbf{x}^{*}
\end{array}\right.
$$

as (25) and (26) at the top of the next page, where $\mathbb{E}[]$ is the expectation operator, $\otimes$ denotes the Kronecker product, $\mathbf{R}_{\operatorname{vec}(\mathbf{H})}=\mathbb{E}\left[\operatorname{vec}(\mathbf{H}) \operatorname{vec}(\mathbf{H})^{H}\right]$, and $P_{\omega}$ is the noise power. The elements of $\mathbf{R}_{\mathrm{vec}(\mathbf{H})}$ are the self and cross correlations of the channel coefficient samples, which can be calculated according to the power spectrum of the channel model.

In the Appendix, we prove that (23) is equivalent to the analytical expression of $F_{\mathrm{Re}, x_{m, n}^{\prime}}(\lambda, \mathbf{x})$ derived using the CDF of $x_{m, n}^{\prime}$ in the Rayleigh fading situation.

The BEP of the 4-PAM signal can then be obtained by (15-17) and (19). In the following section, we will provide the general BEP expressions for PAM signals with different constellation orders.

Remark 1: The expressions above can be greatly simplified for the doubly-flat channels if there is no synchronization error at the receiver. Owing to the absence of ICI and ISI in this case, we do not need to evaluate the interference caused by the other data symbols; thus, the numerical calculation process in (19) is not required. $F_{\mathrm{Re}, x_{m, n}^{\prime}}\left(\lambda, x_{m, n}\right)$ can be calculated directly according to (23) by making the variable substitution: $\mathbf{x} \rightarrow x_{m, n}$. Consequently, (26) is reduced to $\mathbb{E}\left[\left|y_{m, n}\right|^{2} \mid x_{m, n}\right]=\left|x_{m, n}\right|^{2}+P_{\omega}, \mathbb{E}\left[\left|h_{m, n}\right|^{2}\right]=1$, $\mathbb{E}\left[y_{m, n} h_{m, n}^{\prime *} \mid x_{m, n}\right]=x_{m, n}$, and $\mathbb{E}\left[y_{m, n}^{*} h_{m, n}^{\prime} \mid x_{m, n}\right]=x_{m, n}^{*}$, and thus the moment expressions in (25) are given as

$$
\left\{\begin{array}{l}
m_{\alpha}=\sqrt{P_{L O S}} \\
m_{\beta}=\sqrt{P_{L O S}}\left(x_{m, n}-\lambda\right) \\
m_{\alpha \alpha}=1-P_{L O S} \\
m_{\beta \beta}=\left(1-P_{L O S}\right)\left(\left|\left(x_{m, n}\right)\right|^{2}+\lambda^{2}-2 \lambda \Re\left\{x_{m, n}\right\}\right)+P_{\omega} \\
m_{\alpha \beta}=\left(1-P_{L O S}\right)\left(x_{m, n}^{*}-\lambda\right)
\end{array}\right.
$$

\subsection{General BEP Expressions for $O$-Ary PAM Signal}

Now we extend the BEP analysis from 4-PAM to the general case, the $O$-ary PAM signal. The bits of the signal points in PAM constellations with Gray coding are mapped as shown in Table 1. When a data symbol estimated at the receiver is different from the one transmitted, it will cause some specific bit errors. This means we should calculate the error probability of all transmitting-receiving symbol pairs, specify the relationship between the bit error and symbol error, and obtain the BEP accordingly.

A regular pattern of the Gray-coded PAM signal can be observed in Table 1 for the BEP analysis. There are $\log _{2} O$ bits to be represented in a $O$-ary PAM symbol. Let us denote the bit sequences in Table 1 as $b_{1} b_{2} \ldots b_{i} \ldots b_{\log _{2}} O$, i.e., the left-most bit is the first bit. The most important feature of Gray coding is that there is only one different bit between two adjacent signal points. Namely, it can be regarded that each decision boundary in the constellation associates with a specific bit. For example, as presented in (15) and (16), we only consider the boundaries $\lambda=0$ for bit $b_{1}$ and $\lambda=$ $-2 d, 2 d$ for bit $b_{2}$ when we calculate the BEPs of $b_{1}$ and $b_{2}$ for a 4-PAM signal. In addition, we can find that it is a many to one mapping for the decision boundaries and bits. To be specific, there are $2^{i-1}$ decision boundaries associate with the $i$-th bit $b_{i}$, and the distance between two adjacent boundaries among them is $\left(4 O 2^{-i}\right) d$. The relationships of the bits, decision boundaries and distances are shown in Table 2.

Moreover, if the decision statistic $\xi^{\prime}$ is smaller than the left boundaries of the transmitted signal point $\xi$ that is mapped to bit $b_{i}$ in the constellation, from the nearest to the farthest, $b_{i}$ is flipped alternately between the wrong and right 
Table 1 Bit sequences of the PAM symbols using Gray coding [30].

\begin{tabular}{|c|c|c|c|c|c|c|c|c|c|c|c|c|c|c|c|c|}
\hline$O$-ary PAM & \multicolumn{8}{|c|}{ Left Half Plane } & \multicolumn{8}{|c|}{ Right Half Plane } \\
\hline 2-PAM & & & & & & & & 1 & 0 & & & & & & & \\
\hline 4-PAM & & & & & & & 11 & 10 & 00 & 01 & & & & & & \\
\hline 8-PAM & & & & & 111 & 110 & 100 & 101 & 001 & 000 & 010 & 011 & & & & \\
\hline 16-PAM & 1111 & 1110 & 1100 & 1101 & 1001 & 1000 & 1010 & 1011 & 0011 & 0010 & 0000 & 0001 & 0101 & 0100 & 0110 & 0111 \\
\hline$\ldots$ & & & & & & & & & & & & & & & & \\
\hline
\end{tabular}

Table 2 Decision boundaries for bits of PAM signal.

\begin{tabular}{|c|c|c|c|}
\hline$O$-ary PAM & Bit & Decision Boundaries & Distance \\
\hline 2-PAM & $b_{1}$ & 0 & - \\
\hline 4-PAM & $\begin{array}{l}b_{1} \\
b_{2}\end{array}$ & $\begin{array}{c}0 \\
2 d,-2 d\end{array}$ & - \\
\hline 8-PAM & $\begin{array}{l}b_{1} \\
b_{2} \\
b_{3}\end{array}$ & $\begin{array}{c}0 \\
4 d,-4 d \\
-6 d,-2 d, 2 d, 6 d\end{array}$ & $\begin{array}{l}- \\
8 d \\
4 d\end{array}$ \\
\hline 16-PAM & $\begin{array}{l}b_{1} \\
b_{2} \\
b_{3} \\
b_{4}\end{array}$ & $\begin{array}{c}0 \\
8 d,-8 d \\
-12 d,-4 d, 4 d, 12 d \\
-14 d,-10 d,-6 d,-2 d, 2 d, 6 d, 10 d, 14 d\end{array}$ & $\begin{array}{c}- \\
16 d \\
8 d \\
4 d\end{array}$ \\
\hline$\ldots$ & $\ldots$ & $\ldots$ & $\ldots$ \\
\hline
\end{tabular}

values. This is the same rule when $\xi^{\prime}$ is larger than the right boundaries.

For the BEP calculation, owing to the symmetry of the constellation and the equal transmission probabilities of the signal points, we only have to calculate the BEP for the case in which only the signal points located in the right half plane of the constellation are transmitted.

According to the discussion above, the error probability of the $i$-th bit can be determined as follows:

$$
\begin{aligned}
P_{O}(i) & =\frac{1}{O} \sum_{k_{1}=1}^{O} \sum_{k_{2}=1}^{2^{i-1}(-1)}\left\lfloor\frac{\mathfrak{R}\left\{\lambda_{k_{2}}-\xi_{k_{1}} \mid\right\}}{\left(4 O 2^{-i}\right) d}\right\rfloor_{F_{\mathrm{Re}, \xi_{k_{1}}^{\prime}}^{\prime}}\left(\lambda_{k_{2}}, \xi_{k_{1}}\right) \\
& =\frac{2}{O} \sum_{k_{1}=1}^{O / 2} \sum_{k_{2}=1}^{2^{i-1}(-1)}\left\lfloor\frac{\mathfrak{R}\left\{\left|\lambda_{k_{2}-\xi_{k_{1}}}\right|\right\}}{\left(4 O 2^{2}\right) d}\right\rfloor_{F_{\mathrm{Re}, \xi_{k_{1}}^{\prime}}^{\prime}}\left(\lambda_{k_{2}}, \xi_{k_{1}}\right)
\end{aligned}
$$

where $\xi_{k_{1}}=\left(O-2 k_{1}+1\right) d$ is the $k_{1}$-th transmitted signal point, $\xi_{k_{1}}^{\prime}$ is the corresponding received decision statistic, $\lambda_{k_{2}}=\left(-O+2 O 2^{-i}+4 O\left(k_{2}-1\right) 2^{-i}\right) d$ is the $k_{2}$-th decision boundary, and

$$
F_{\mathrm{Re}, \xi^{\prime}}^{\prime}(\lambda, \xi)= \begin{cases}F_{\mathrm{Re}, \xi^{\prime}}(\lambda, \xi) & \text { if } \lambda<\xi \\ 1-F_{\mathrm{Re}, \xi^{\prime}}(\lambda, \xi) & \text { if } \lambda \geq \xi\end{cases}
$$

where $F_{\mathrm{Re}, \xi^{\prime}}(\lambda, \xi)$ is the conditional probability function defined in (18). Finally, the exact expression of the average BEP of a $O$-ary PAM signal can be obtained by averaging the BEP given in (28):

$$
P_{b, O-P A M}=\frac{1}{\log _{2} O} \sum_{i=1}^{\log _{2} O} P_{O}(i)
$$

\subsection{BEP Evaluation for QAM Signal}

As mentioned earlier, a square QAM signal can be regarded as a combination of two independent in-phase and quadrature PAM signals. Thus the BEP expression for $O$-ary square QAM is similar to that for a $\sqrt{O}$-ary PAM signal:

$$
P_{b, O-Q A M}=P_{b, \sqrt{O}-P A M}=\frac{1}{\log _{2} \sqrt{O}} \sum_{i=1}^{\log _{2} \sqrt{O}} P_{\sqrt{O}}(i)
$$

where $P_{\sqrt{O}}(i)$ is the $i$-th BEP determined in (28). However, different from the case of the PAM signal, the QAM data symbols $\xi_{k_{1}^{\prime}}$ used to perform calculations in (28) are complex and can be a arbitrary group of symbols located in the inphase dimension, e.g., $\xi_{k_{1}}=\left(O-2 k_{1}+1\right) d+j d$.

\section{Simulation}

In this section, we present the simulation results in order to validate the previous theoretical analysis. We consider the FBMC-OQAM system and two OFDM variants: CPOFDM and non-CP-OFDM. The subcarrier bandwidth is set as $F=15 \mathrm{kHz}$, and the length of the $\mathrm{CP}$ adopted in $\mathrm{CP}$ OFDM equals $T / 14$ of the non-CP-OFDM, which are the same as those in LTE. For a fair comparison, the symbol pe$\operatorname{riod} T$ of FBMC-OQAM is half of non-CP-OFDM, and the order of PAM adopted in FBMC-OQAM is the square root of the order of QAM in OFDM. Thus, FBMC-OQAM achieves the same data rate as non-CP-OFDM. We employ the Hermite filter in the FBMC-OQAM system, and the overlapping factor is set to 6 . In order to exactly evaluate the effects of ICI and ISI in both OFDM and FBMC-OQAM systems with multipath fading channels, a sufficient number of subcarriers and symbols must be considered. In the simulation, $M=200$ and $N=7$ are selected. The sampling rate is $15 \mathrm{MHz}$, and we consider only the data symbol located in the middle position among all subcarriers and symbols to evaluate the BEP performance accurately.

Figure 2 presents the BEP performances of OFDM and FBMC-OQAM systems in Rayleigh and Rician flat channels calculated based on the analytical expressions derived in this paper and in [9]. It can be observed that the numerical simulation results agree well with the theoretical results calculated for different modulation constellation sizes. The performances of non-CP-OFDM, CP-OFDM and FBMCOQAM systems are also similar. This is mainly owing to the absence of ICI and ISI in the flat channel.

Agreements between the results of theoretical analysis and the numerical simulation can also be observed in doublyselective channels as shown in Fig. 3. Three types of doublyselective fading channels in [26] are considered: Pedestrian A (Ped-A), Vehicular A (Veh-A), and satellite A (Sat-A). In 


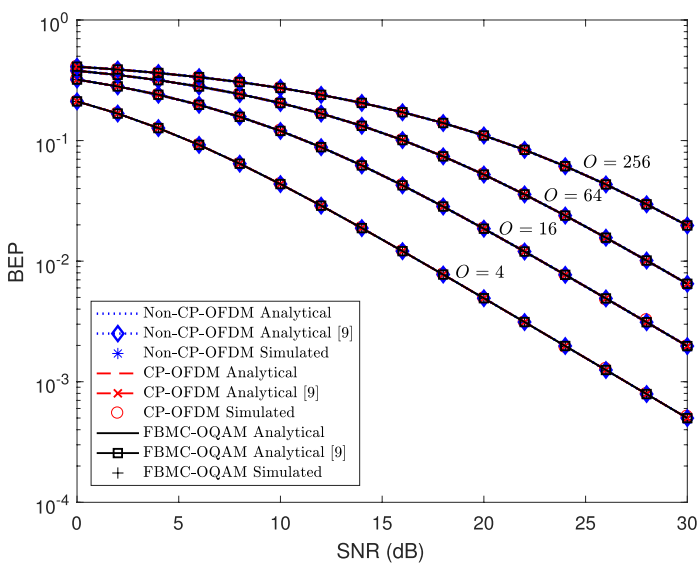

(a) Rayleigh Flat Channel

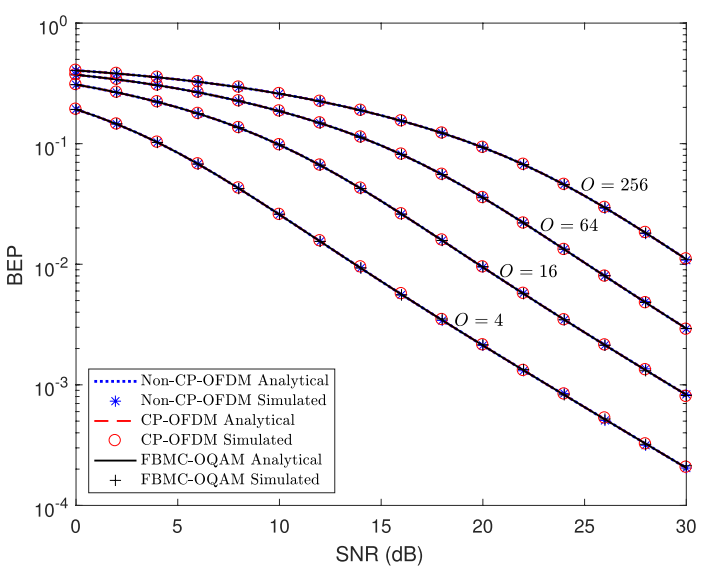

(b) Rician Flat Channel, Rician factor $=3 \mathrm{~dB}$

Fig. 2 The BEP performances of $O$-ary QAM for OFDM systems and $\sqrt{O}$-ary PAM for FBMC-OQAM system in Rayleigh and Rician doubly-flat channels.

the case of Ped-A and Veh-A channels, the non-CP-OFDM system suffers a greater deterioration in performance than the CP-OFDM and FBMC-OQAM systems because of the presence of ICI and ISI. Because both the velocity of the receiver and the interfering multipath power are assumed to be very low in the Ped-A channel, the Doppler spread and delay spread are small, and thus the BEP performances of FBMC-OQAM and CP-OFDM systems stay almost the same over a large range of SNRs in the Ped-A channel. This also explains the results of the Sat-A channel, where the Doppler shift caused by the movement of the satellite is assumed to be compensated perfectly. In addition to the small time and frequency spreads, there is an LOS path in the Sat-A channel that results in better BEP performance than in the Ped-A channel. However, in the harsher Veh-A channel, the large Doppler spread makes ICI the dominant constraint at high SNR values, and therefore the FBMC-OQAM system outperforms the CP-OFDM system.

The effects of the synchronization errors can also be evaluated. Figure 4 shows the BEP performances in the presence of CFO and TO. As shown in Fig. 4, the FBMC-OQAM

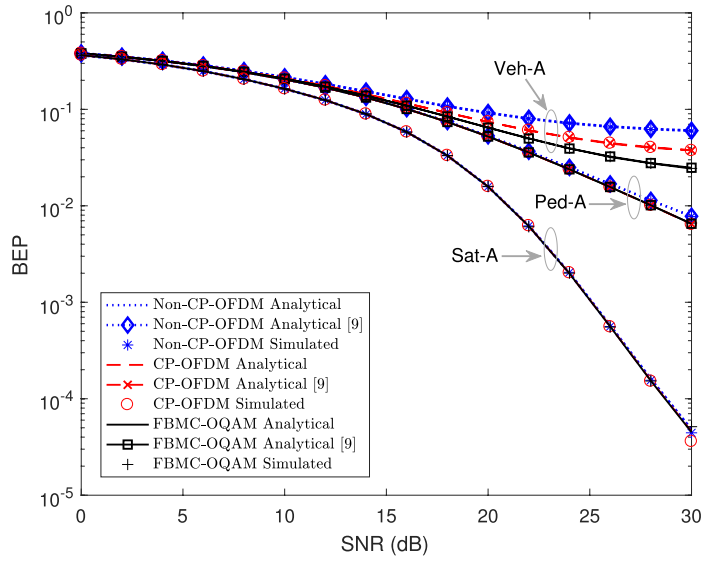

Fig. 3 BEP performances of 64-QAM for OFDM systems and 8-PAM for FBMC-OQAM system in three ITU doubly-selective channels: Ped-A (Rayleigh) at $3 \mathrm{~km} / \mathrm{h}$, Veh-A (Rayleigh) at $500 \mathrm{~km} / \mathrm{h}$, and Sat-A (Rician) at $70 \mathrm{~km} / \mathrm{h}$.

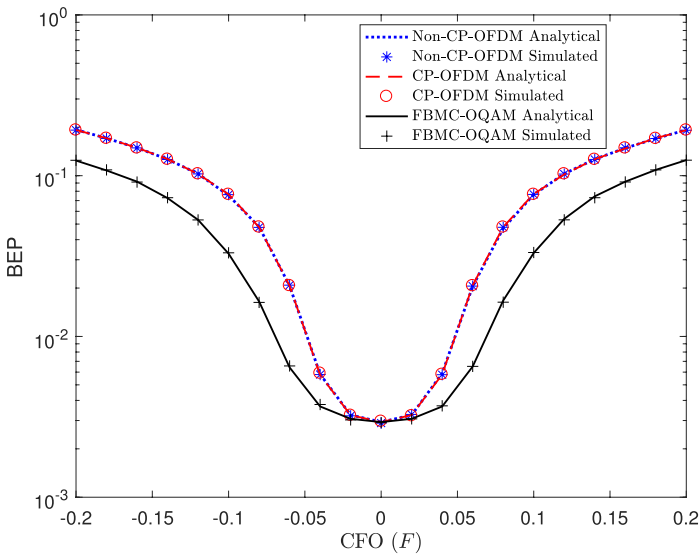

(a) $\mathrm{CFO}$

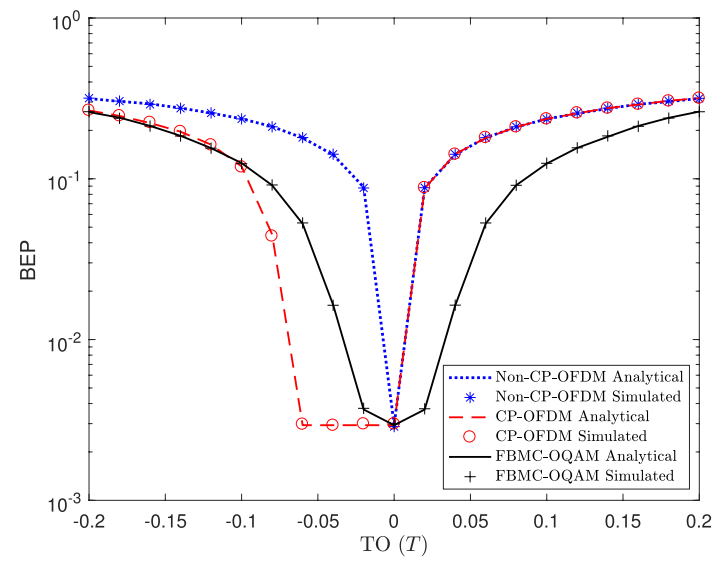

(b) $\mathrm{TO}$

Fig. 4 BEP performances of OFDM systems and FBMC-OQAM system in the Rician flat channel in the case of existing $\mathrm{CFO}$ and TO, and Rician factor $=3 \mathrm{~dB}$. In simulations of OFDM and FBMC-OQAM systems, 64$\mathrm{QAM}$ and 8-PAM at $\mathrm{SNR}=30 \mathrm{~dB}$ are used, respectively. Range of TO and CFO extends from zero to $\pm 0.2 T$ and $\pm 0.2 F$, respectively. 
system is less sensitive to CFO than the OFDM systems, owing to its filter with excellent TFL property. In the case of the timing offset, because the $\mathrm{CP}$ interval introduces a time zone that can absorb the timing offset, there is a negligible effect on CP-OFDM when $\mathrm{TO}$ is within the $\mathrm{CP}$ period range. If $\mathrm{TO}$ exceeds the $\mathrm{CP}$-protected time range, the performance of the CP-OFDM system deteriorates drastically as the nonCP-OFDM system, which is also more sensitive to TO than the FBMC-OQAM system. In fact, by inserting a protect subcarrier between the bands occupied by the adjacent users to against different TOs and CFOs, the FBMC-OQAM system can achieve much better performance than the OFDM system and is thus considered a promising efficient solution for asynchronous multiuser communication scenarios [11], [12], [31].

\section{Conclusion and Future Works}

In this paper, we have presented a general analytical framework for evaluating the basic BEP performances of FBMCOQAM and OFDM systems. By virtue of this analytical framework, different channel cases including Rayleigh and Rician fading, and flat and selective channels can be considered. We have also derived the exact closed-form BEP expressions for Gray-coded PAM and square QAM signals with arbitrary constellation sizes. The accuracy of the theoretical analysis has been validated by simulation results. According to the simulation results, the FBMC-OQAM system achieves the same BEP performances as OFDM in flat channels and even better performances than CP-OFDM in highly time-varying channels, while maintaining higher frequency spectral efficiency. In addition, the proposed analytical framework can easily be extended to evaluate the BEP performances of FBMC-OQAM and OFDM systems in asynchronous communication scenarios.

For future work, more practical issues, e.g., channel estimation error, phase noise, and nonlinear distortion, can be taken into consideration. Besides, given that channel coding is widely used in communication systems to protect data symbols from noise and interference, the analytical BEP evaluation of coded OFDM and FBMC-OQAM signals needs to be addressed.

\section{References}

[1] B. Farhang-Boroujeny, "OFDM versus filter bank multicarrier," IEEE Signal Process. Mag., vol.28, no.3, pp.92-112, 2011.

[2] C. Sexton, Q. Bodinier, A. Farhang, N. Marchetti, F. Bader, and L.A. DaSilva, "Enabling asynchronous machine-type D2D communication using multiple waveforms in 5G," IEEE Internet Things J., vol.5, no.2, pp.1307-1322, April 2018.

[3] H. Saeedi-Sourck, "Multiple carrier frequency offsets and multipath channels estimation for coordinated multi-point FBMC systems," Phys. Commun., vol.25, pp.511-518, Dec. 2017.

[4] H. Zhang, D. Le Ruyet, D. Roviras, Y. Medjahdi, and H. Sun, "Spectral efficiency comparison of OFDM/FBMC for uplink cognitive radio networks," EURASIP J. Adv. Signal Process., vol.2010, pp.4:1-4:11, Jan. 2010

[5] C. Sexton, Q. Bodinier, A. Farhang, N. Marchetti, F. Bader, and
L.A. DaSilva, "Coexistence of OFDM and FBMC for underlay D2D communication in 5G networks," 2016 IEEE Globecom Workshops (GC Wkshps), pp.1-7, Dec. 2016.

[6] A. Sahin, I. Guvenc, and H. Arslan, "A survey on multicarrier communications: Prototype filters, lattice structures, and implementation aspects," IEEE Commun. Surveys Tuts., vol.16, no.3, pp.1312-1338, 2014

[7] H. Lin, M. Gharba, and P. Siohan, "Impact of time and carrier frequency offsets on the FBMC/OQAM modulation scheme," Signal Process., vol.102, no.Supplement C, pp.151-162, Sept. 2014.

[8] R. Nissel, S. Schwarz, and M. Rupp, "Filter bank multicarrier modulation schemes for future mobile communications," IEEE J. Sel. Areas Commun., vol.35, no.8, pp.1768-1782, Aug. 2017.

[9] R. Nissel and M. Rupp, "OFDM and FBMC-OQAM in doublyselective channels: Calculating the bit error probability," IEEE Commun. Lett., vol.21, no.6, pp.1297-1300, June 2017.

[10] D. Gregoratti and X. Mestre, "Uplink FBMC/OQAM-based multiple access channel: Distortion analysis under strong frequency selectivity,” IEEE Trans. Signal Process., vol.64, no.16, pp.4260-4272, 2016.

[11] H. Saeedi-Sourck, Y. Wu, J.W.M. Bergmans, S. Sadri, and B. Farhang-Boroujeny, "Complexity and performance comparison of filter bank multicarrier and OFDM in uplink of multicarrier multiple access networks," IEEE Trans. Signal Process., vol.59, no.4, pp.1907-1912, April 2011.

[12] D. Mattera, M. Tanda, and M. Bellanger, "Performance analysis of some timing offset equalizers for FBMC/OQAM systems," Signal Process., vol.108, pp.167-182, 2015.

[13] S. Dimitrov, N. Privitera, R. Suffritti, G. Boccolini, A.B. Awoseyila, and B.G. Evans, "Spectrally efficient waveforms for the return link in satellite communication systems," 2015 European Conference on Networks and Communications (EuCNC), pp.6-10, June 2015.

[14] Y. Medjahdi, M. Terre, D.L. Ruyet, D. Roviras, and A. Dziri, "Performance analysis in the downlink of asynchronous OFDM/FBMC based multi-cellular networks," IEEE Trans. Wireless Commun., vol.10, no.8, pp.2630-2639, 2011.

[15] H. Bouhadda, H. Shaiek, D. Roviras, R. Zayani, Y. Medjahdi, and R. Bouallegue, "Theoretical analysis of BER performance of nonlinearly amplified FBMC/OQAM and OFDM signals," EURASIP J. Adv. Signal Process., vol.2014, p.60, May 2014.

[16] B. Elmaroud, A. Faqihi, and D. Aboutajdine, "Sensitivity analysis of FBMC-based multi-cellular networks to synchronization errors and HPA nonlinearities," EURASIP J. Adv. Signal Process., vol.2017, p.3, Jan. 2017.

[17] R. Nissel and M. Rupp, "Bit error probability for pilot-symbol aided channel estimation in FBMC-OQAM," 2016 IEEE International Conference on Communications (ICC), pp.1-6, May 2016.

[18] M. Sriyananda, "Analysis and performance of FBMC techniques with application to relay networks," Jyvaskyla Studies in Computing, no. 199,2014

[19] H. Murata, E. Okamoto, M. Mikami, A. Okazaki, S. Suyama, T. Inoue, J. Mashino, T. Yamamoto, and M. Taromaru, "Radio access technologies for fifth generation mobile communications system: Review of recent research and developments in Japan," IEICE Trans. Commun., vol.E99-B, no.8, pp.1638-1647, Aug. 2016.

[20] X. Cheng, Y. Li, B. Ai, X. Yin, and Q. Wang, "Device-to-device channel measurements and models: A survey," IET Commun., vol.9, no.3, pp.312-325, Jan. 2015.

[21] M. Peng, Y. Li, T.Q.S. Quek, and C. Wang, "Device-to-device underlaid cellular networks under rician fading channels," IEEE Trans. Wireless Commun., vol.13, no.8, pp.4247-4259, Aug. 2014.

[22] S. Mirabbasi and K. Martin, "Overlapped complex-modulated transmultiplexer filters with simplified design and superior stopbands," IEEE Trans. Circuits Syst. II, Exp. Briefs, vol.50, no.8, pp.456-469, Aug. 2003.

[23] M. Bellanger, D. Le Ruyet, D. Roviras, M. Terré, J. Nossek, L. Baltar, Q. Bai, D. Waldhauser, M. Renfors, and T. Ihalainen, "FBMC phys- 
ical layer: A primer," PHYDYAS, vol.25, no.4, pp.7-10, Jan. 2010.

[24] R. Haas and J.C. Belfiore, "A time-frequency well-localized pulse for multiple carrier transmission," Wirel. Pers. Commun., vol.5, no.1, pp.1-18, 1997.

[25] B.L. Floch, M. Alard, and C. Berrou, "Coded orthogonal frequency division multiplex," Proc. IEEE, vol.83, no.6, pp.982-996, June 1995.

[26] ITU, Recommendation ITU-R M.1225: Guidelines for evaluation of radio transmission technologies for IMT-2000, Technical Report, ITU, 1997.

[27] R.J. Baxley, B.T. Walkenhorst, and G. Acosta-Marum, "Complex Gaussian ratio distribution with applications for error rate calculation in fading channels with imperfect CSI," 2010 IEEE Global Telecommunications Conference GLOBECOM 2010, pp.1-5, Dec. 2010.

[28] J. Proakis, "On the probability of error for multichannel reception of binary signals," IEEE Trans. Commun. Technol., vol.16, no.1, pp.68-71, 1968.

[29] P. Tan and N.C. Beaulieu, "Effect of channel estimation error on bit error probability in OFDM systems over Rayleigh and Ricean fading channels," IEEE Trans. Commun., vol.56, no.4, pp.675-685, 2008.

[30] C. Kyongkuk and Y. Dongweon, "On the general BER expression of one- and two-dimensional amplitude modulations," IEEE Trans. Commun., vol.50, no.7, pp.1074-1080, 2002.

[31] J.B. Dore, V. Berg, and D. Ktenas, "Performance of FBMC multiple access for relaxed synchronization cellular networks," 2014 IEEE Globecom Workshops (GC Wkshps), pp.983-988, Dec. 2014.

[32] M. Alouini and A.J. Goldsmith, "A unified approach for calculating error rates of linearly modulated signals over generalized fading channels," IEEE Trans. Commun., vol.47, no.9, pp.1324-1334, Sept. 1999.

\section{Appendix: Conditional CDF of $\mathfrak{R}\left\{x_{m, n}^{\prime}\right\}$ over Rayleigh Fading Channels}

In this Appendix we will show that the expression of $F_{\mathrm{Re}, x_{m, n}^{\prime}}(\lambda, \mathbf{x})$ given by (23) is essentially the same as that derived according to the $\mathrm{CDF}$ of the complex ratio distribution in Rayleigh fading channels. As a special case, the wellknown BEP expression of BPSK signal over the Rayleigh flat channel is also derived.

In the case of Rayleigh channels, $y_{m, n}$ and $h_{m, n}^{\prime}$ can be regarded as zero mean, correlated, complex-valued, Gaussian random variables [9]. The conditional CDF of the complex ratio $x_{m, n}^{\prime}=y_{m, n} / h_{m, n}^{\prime}$ can be obtained as $[9$, Lemma 1]

$$
\begin{aligned}
& F_{x_{m, n}^{\prime}}\left(z_{\mathrm{R}}, z_{\mathrm{I}}, \mathbf{x}\right) \\
= & \operatorname{Pr}\left\{\left(\mathfrak{R}\left\{\frac{y_{m, n}}{h_{m, n}^{\prime}}\right\}<z_{\mathrm{R}}\right) \wedge\left(\mathfrak{I}\left\{\frac{y_{m, n}}{h_{m, n}^{\prime}}\right\}<z_{\mathrm{I}}\right) \mid \mathbf{x}\right\} \\
= & \frac{1}{4}+\frac{\left(z_{\mathrm{R}}-\mathfrak{R}\{\gamma\}\right)\left(2 \tan ^{-1}\left(\frac{z_{\mathrm{I}}-\mathfrak{I}\{\gamma\}}{\sqrt{\left(z_{\mathrm{R}}-\mathfrak{R}\{\gamma\}\right)^{2}+\eta-|\gamma|^{2}}}\right)+\pi\right)}{4 \pi \sqrt{\left(z_{\mathrm{R}}-\mathfrak{R}\{\gamma\}\right)^{2}+\eta-|\gamma|^{2}}} \\
+ & \frac{\left(z_{\mathrm{I}}-\mathfrak{I}\{\gamma\}\right)\left(2 \tan ^{-1}\left(\frac{z_{\mathrm{R}}-\mathfrak{R}\{\gamma\}}{\sqrt{\left(z_{\mathrm{I}}-\mathfrak{I}\{\gamma\}\right)^{2}+\eta-|\gamma|^{2}}}\right)+\pi\right)}{4 \pi \sqrt{\left(z_{\mathrm{I}}-\mathfrak{I}\{\gamma\}\right)^{2}+\eta-|\gamma|^{2}}},
\end{aligned}
$$

where

$$
\gamma=\frac{\mathbb{E}\left[y_{m, n} h_{m, n}^{\prime *} \mid \mathbf{x}\right]}{\mathbb{E}\left[\left|h_{m, n}^{\prime}\right|^{2}\right]} \text { and } \eta=\frac{\mathbb{E}\left[\left|y_{m, n}\right|^{2} \mid \mathbf{x}\right]}{\mathbb{E}\left[\left|h_{m, n}^{\prime}\right|^{2}\right]} .
$$

Then $F_{\mathrm{Re}, x_{m, n}^{\prime}}(\lambda, \mathbf{x})$ can be obtained by setting $z_{\mathrm{R}}$ and $z_{\mathrm{I}}$ to $\lambda$ and $\infty$ respectively:

$$
\begin{aligned}
& F_{\mathrm{Re}, x_{m, n}^{\prime}}(\lambda, \mathbf{x})=F_{x_{m, n}^{\prime}}(\lambda, \infty, \mathbf{x}) \\
& =\frac{1}{4}+\frac{2 \tan ^{-1}(0)+\pi}{4 \pi}+\frac{(\lambda-\mathfrak{R}\{\gamma\})\left(2 \tan ^{-1}(\infty)+\pi\right)}{4 \pi \sqrt{(\lambda-\mathfrak{R}\{\gamma\})^{2}+\eta-|\gamma|^{2}}} \\
& \left.=\frac{1}{2}+\operatorname{sgn}(\lambda-\mathfrak{R}\{\gamma\})\right) \frac{1}{2 \sqrt{1+\frac{\eta-|\gamma|^{2}}{(\lambda-\mathfrak{R}\{\gamma\})^{2}}}} \quad \text { (A. 3) }
\end{aligned}
$$

where $\operatorname{sgn}(\cdot)$ is the sign function.

Alternatively, we can use Eq. (23) to calculate $F_{\mathrm{Re}, x_{m, n}^{\prime}}(\lambda, \mathbf{x})$. For a Rayleigh channel, $P_{L O S}=0$, and then $m_{\alpha}$ and $m_{\beta}$ in (25) are zero. The values of $a, b, u_{1}$, and $u_{2}$ in (24) also become 0 accordingly. Then, (23) is simplified to

$$
\begin{aligned}
& F_{\mathrm{Re}, x_{m, n}^{\prime}}(\lambda, \mathbf{x})=1-\frac{v_{2}}{v_{1}+v_{2}} \\
& =1-\frac{\sqrt{\omega^{2}+4 /\left(m_{\alpha \alpha} m_{\beta \beta}-\left|m_{\alpha \beta}\right|^{2}\right)}+\omega}{2 \sqrt{\omega^{2}+4 /\left(m_{\alpha \alpha} m_{\beta \beta}-\left|m_{\alpha \beta}\right|^{2}\right)}} \\
& =\frac{1}{2}-\operatorname{sgn}(\omega) \frac{1}{2 \sqrt{1+\frac{4}{\left(m_{\alpha \alpha} m_{\beta \beta}-\left|m_{\alpha \beta}\right|^{2}\right) \omega^{2}}}}
\end{aligned}
$$

where $\left(m_{\alpha \alpha} m_{\beta \beta}-\left|m_{\alpha \beta}\right|^{2}\right) \omega^{2}$ and $\operatorname{sgn}(\omega)$ can be written as (A. 5) and (A.6) respectively, shown at the top of this page, and we omit the subscript $(m, n)$ and conditional notation $\mid \mathbf{x}$ in (A. 5) for brevity. Substituting (A. 5) and (A. 6) into (A. 4), we can find that (A.4) and (A. 3) get the same result.

In the case of Rayleigh doubly-flat fading channel, according to (28) and (30), the well-known BEP expression for the BPSK signal [32] can be obtained as

$$
\begin{aligned}
P_{b, \mathrm{BPSK}} & =P_{2}(1) \\
& =F_{\mathrm{Re}, \xi^{\prime}=d}(0, d) \\
& =\frac{1}{2}-\frac{1}{2 \sqrt{1+\frac{p_{\omega}}{|d|^{2}}}} \\
& =\frac{1}{2}-\frac{1}{2} \sqrt{\frac{\mathrm{SNR}}{1+\mathrm{SNR}}}
\end{aligned}
$$

where $d^{2}$ denotes the power of the BPSK signal, and SNR = $d^{2} / P_{\omega}$ denotes the symbol SNR. 


$$
\begin{aligned}
& \frac{4}{\left(m_{\alpha \alpha} m_{\beta \beta}-\left|m_{\alpha \beta}\right|^{2}\right) \omega^{2}}=\frac{m_{\alpha \alpha} m_{\beta \beta}-\left|m_{\alpha \beta}\right|^{2}}{\left(\mathfrak{R}\left\{m_{\alpha \beta}\right\}\right)^{2}}=\frac{\mathbb{E}\left[\left|h^{\prime}\right|^{2}\right] \mathbb{E}\left[\left|y^{\prime}+\lambda h^{\prime}\right|^{2}\right]-\left|\mathbb{E}\left[y^{*} h^{\prime}\right]-\lambda \mathbb{E}\left[\left|h^{\prime}\right|^{2}\right]\right|^{2}}{\left(\mathfrak{R}\left\{\mathbb{E}\left[y^{*} h^{\prime}\right]-\lambda \mathbb{E}\left[\left|h^{\prime}\right|^{2}\right]\right\}\right)^{2}} \\
& =\frac{\mathbb{E}\left[\left|h^{\prime}\right|^{2}\right] \mathbb{E}\left[|y|^{2}\right]+\lambda^{2}\left(\mathbb{E}\left[\left|h^{\prime}\right|^{2}\right]\right)^{2}-2 \lambda \mathbb{E}\left[\left|h^{\prime}\right|^{2}\right] \mathfrak{R}\left\{\mathbb{E}\left[y^{*} h^{\prime}\right]\right\}-\left(\left|\mathbb{E}\left[y^{*} h^{\prime}\right]\right|^{2}-2 \lambda \mathbb{E}\left[\left|h^{\prime}\right|^{2}\right] \mathfrak{R}\left\{\mathbb{E}\left[y^{*} h^{\prime}\right]\right\}+\lambda^{2}\left(\mathbb{E}\left[\left|h^{\prime}\right|^{2}\right]\right)^{2}\right)}{\left(\mathfrak{R}\left\{\mathbb{E}\left[y^{*} h^{\prime}\right]-\lambda \mathbb{E}\left[\left|h^{\prime}\right|^{2}\right]\right\}\right)^{2}} \\
& =\frac{\mathbb{E}\left[\left|h^{\prime}\right|^{2}\right] \mathbb{E}\left[|y|^{2}\right]-\left|\mathbb{E}\left[y^{*} h^{\prime}\right]\right|^{2}}{\left(\mathfrak{R}\left\{\mathbb{E}\left[y^{*} h^{\prime}\right]-\lambda \mathbb{E}\left[\left|h^{\prime}\right|^{2}\right]\right\}\right)^{2}}=\frac{\mathbb{E}\left[|y|^{2}\right] / \mathbb{E}\left[\left|h^{\prime}\right|^{2}\right]-\left|\mathbb{E}\left[y^{*} h^{\prime}\right] / \mathbb{E}\left[\left|h^{\prime}\right|^{2}\right]\right|^{2}}{\left(\mathfrak{R}\left\{\mathbb{E}\left[y^{*} h^{\prime}\right] / \mathbb{E}\left[\left|h^{\prime}\right|^{2}\right]-\lambda\right\}\right)^{2}}=\frac{\eta-|\gamma|^{2}}{(\lambda-\mathfrak{R}\{\gamma\})^{2}} \\
& \operatorname{sgn}(\omega)=\operatorname{sgn}\left(\frac{2 \mathfrak{R}\left\{m_{\alpha \beta}\right\}}{\left(m_{\alpha \alpha} m_{\beta \beta}-\left|m_{\alpha \beta}\right|^{2}\right)}\right)=\operatorname{sgn}\left(\mathfrak{R}\left\{m_{\alpha \beta}\right\}\right)=\operatorname{sgn}\left(\mathfrak{R}\left\{\mathbb{E}\left[y^{*} h^{\prime}\right]-\lambda \mathbb{E}\left[\left|h^{\prime}\right|^{2}\right]\right\}\right)=\operatorname{sgn}\left(\mathfrak{R}\left\{\frac{\mathbb{E}\left[y h^{\prime *}\right]}{\mathbb{E}\left[\left|h^{\prime}\right|^{2}\right]}-\lambda\right\}\right) \\
& =-\operatorname{sgn}(\lambda-\mathfrak{R}\{\gamma\})
\end{aligned}
$$

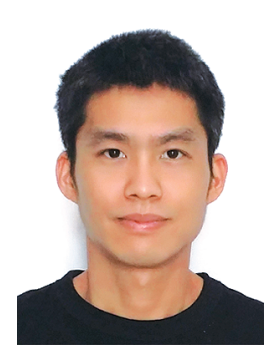

Liming $\mathbf{L i}$ received the B.S. degree in Communication Engineering from Shandong University, China, in 2007, and the M.S. degree in Biomedical Engineering from Chongqing University, China, in 2013. He is currently pursuing the Ph.D. degree in Information and Communication Engineering at Shenzhen Graduate School, Harbin Institute of Technology, China. His research interests include multicarrier modulation, synchronization and digital signal processing.

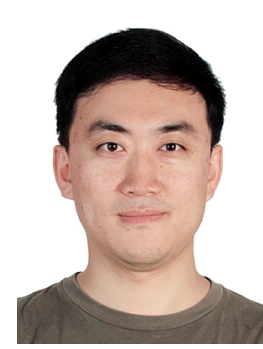

Yang Wang received his Ph.D. degree from Harbin Institute of Technology in 2005 . From 2005 to 2007, he was a postdoctoral at Harbin Institute of Technology Shenzhen Graduate School, Shenzhen, China. He is currently an associate professor and doctoral supervisor of Harbin Institute of Technology Shenzhen Graduate School. He is a senior member of Chinese Institute of Electronics. His research interests include wireless communications, UWB system, MIMO system, signal processing and coopera-

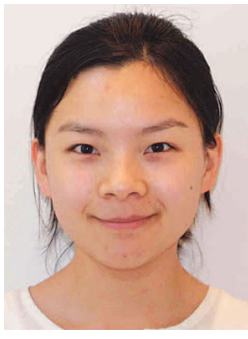

Liqin Ding received the B.S., M.S. and Ph.D. degrees in Information and Communication Engineering from Harbin Institute of Technology, China, in 2009, 2011 and 2017, respectively. She was visiting the Department of Electronics and Telecommunications, Norwegian University of Science and Technology from Sept. 2012 to Sept. 2014. She is currently a postdoctoral at Shenzhen Graduate School, Harbin Institute of Technology, China. Her research interests include lattice theory, signal processing and cooperative design for wireless communications.

tive communications. 